\title{
Photonic-assisted microwave frequency measurement towards cognitive radio
}

\section{Medição de frequência de micro-ondas utilizando técnicas fotônicas rumo ao rádio cognitivo}

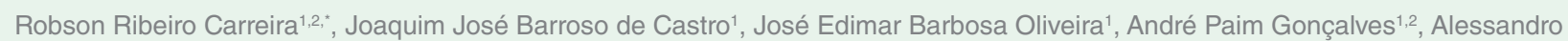
Roberto dos Santos ${ }^{1,2}$

\begin{abstract}
Cognitive radio frequency (RF) systems can be seen through an OODA (observe, orient, decide and act) loop, and electronic warfare (EW) can be used in some of these stages, namely the observe and the act. Instantaneous microwave frequency measurement (IFM) systems can contribute to cognitive RF technology taking the real world to the data world. In an OODA loop, IFM can be arranged as in the observe stage. Here we have proposed an IFM device that allows the adjustment of the directional couplers coupling coefficients, optical fiber lengths, modulation index and modulation formats, which makes the system tunable. The analytical model result shows good agreement with the simulation, achieving frequency differences of $130 \mathrm{MHz}$ and $210 \mathrm{MHz}$ for the lowest and the highest amplitude comparison function values, respectively.
\end{abstract}

Keywords: Electronic warfare, Instantaneous frequency measurement, Amplitude comparison function.

\section{RESUMO}

Os sistemas de radiofrequência cognitivos podem ser vistos por meio de um ciclo OODA (observar, orientar, decidir e agir). Nesse ciclo, a guerra eletrônica pode ser utilizada nas fases da observação e ação. Os sistemas de medição de frequência instantânea de microondas (IFM) podem contribuir para a tecnologia de rádio cognitivo, levando o mundo real para o mundo dos dados. Em um ciclo OODA, o IFM pode ser aplicado na fase da observação. Neste trabalho, propomos um dispositivo IFM que permite o ajuste dos coeficientes de acoplamento dos acopladores direcionais, comprimentos das fibras ópticas, índice de modulação e formatos de modulação, o que torna o sistema sintonizável. O resultado do modelo analítico apresenta boa concordância com a simulação, alcançando diferenças de frequência de $130 \mathrm{MHz}$ e $210 \mathrm{MHz}$ para os valores mais baixos e mais altos da função de comparação de amplitude, respectivamente.

Palavras-chave: Guerra eletrônica, Medição de frequência instantânea, Função comparadora de amplitude.

\footnotetext{
1. Departamento de Ciência e Tecnologia Aeroespacial - Instituto Tecnológico de Aeronáutica - Divisão de Engenharia Eletrônica - São José dos Campos (SP), Brazil.
}

2. Centro de Coordenação de Estudos da Marinha - Departamento de Ensino - São Paulo (SP), Brazil. 


\section{INTRODUCTION}

Cognitive radio technology has been considered a way to perform a spectrum management due to its abilities to learn, infer and react to the environment. Cognitive radio frequency (RF) systems can be described as an OODA (observe, orient, decide and act) loop, as shown in Fig. 1. Firstly, the electromagnetic spectrum is observed through a sensor. Then, the information is processed and oriented, enabling a decision making. Finally, it becomes an act when configuring the transceiver in a proper way ${ }^{1}$.

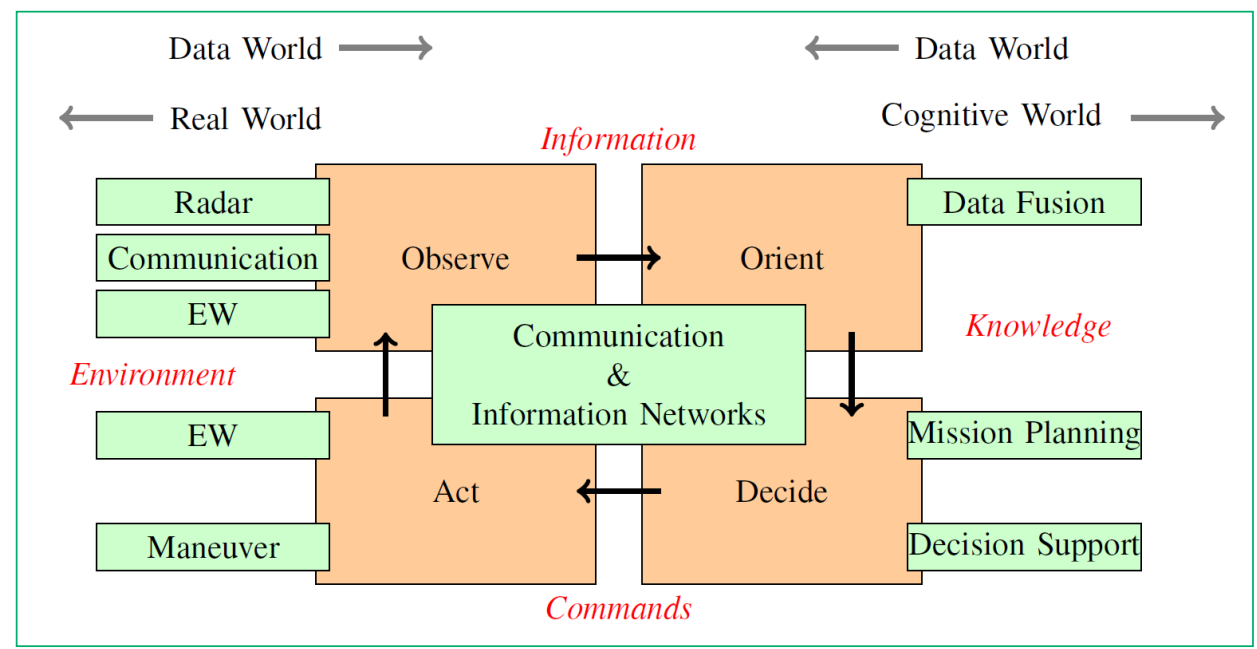

Figure 1: Cognitive radio frequency systems viewed as an OODA (observe, orient, act and decide) loop ${ }^{1}$. The proposed instantaneous microwave frequency measurement system is included in the electronic warfare block, connecting the real world to the data world and feeding the observe block.

Electronic warfare (EW) is the only discipline operating in the electromagnetic spectrum in the observe and the act stages ${ }^{1}$. An available frequency operating range has become scarce, due to the increasingly demand of wireless devices and electromagnetic systems. Therefore, a spectrum management implementation can make RF systems work in a complex electromagnetic environment in a more effective way1. In this sense, instantaneous microwave frequency measurement (IFM) systems can contribute to cognitive RF technology, taking the real world to the data world. In an OODA loop, IFM can be arranged as in the observe stage.

IFM has been used in EW applications, especially electronic support measures (ESM), for over 60 years ${ }^{2}$. Recently, microwave EW industry reached the technology maturity and, thenceforth, applications based on microwave-photonics (MP) have emerged, the socalled photonic-assisted IFM². Nowadays, authors have proposed several architectures aiming at IFM applications ${ }^{3-6}$.

Here, we have proposed a functional block for electromagnetic sensing to provide data acquisition for a radio cognitive system. This approach allows representation in both time and frequency domains, besides offering more flexibility to set up areater number of parameters, namely directional couplers (DC) coefficients, high modulation indexes, and amplitude comparison function (ACF) for double-sideband (DSB) and single-sideband (SSB) modulation formats. This tunable system can be overviewed by a semi-analytical model specifically developed that includes higher order lateral bands, enabling instantaneous ACF ratios for spectral components of orders different than unity ${ }^{7}$

\section{OPERATION PRINCIPLE}

A generic photonic-assisted IFM system architecture can be represented by the block diagram shown in Fig. 2. It consists of a light source, an electrical-to-optical conversion module, a photonic processing module, an optical-to-electrical conversion module, and a post-processing module.

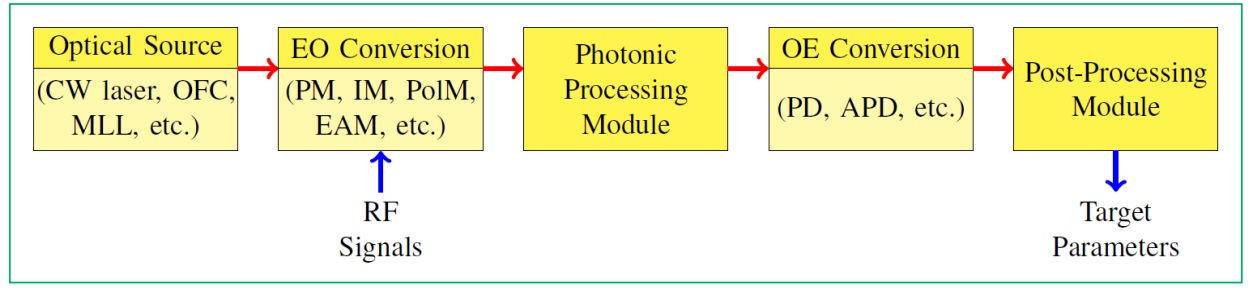

Figure 2: Generic photonic-assisted instantaneous microwave frequency measurement system architecture block diagram ${ }^{8}$.

CW: continuous wave; OFC: optical frequency comb; MLL: Mode-Locked Laser; EO: electro-optic; PM: phase modulator; IM: intensity modulator; PoIM: polarization modulator; EAM: electro-absorption modulator; OE: optical-electro; PD: Photodetector; APD: avalanche photodetector; RF: radio frequency. 
The functional block diagram proposed is shown in Fig. 3. When correlating it with the generic photonic-assisted IFM system architecture from Fig. 2, the laser is the optical source, the dual-output dual-drive Mach-Zehnder modulator (DD-MZM) is the electrical-to-optical conversion module, and the two distinct standard single-mode fiber (SSMF), with lengths $\mathrm{L}_{1}$ and $\mathrm{L}_{2}$, are the photonic processing module, as they perform the desirable chromatic dispersion (CD) effect, which gives rises to the notch regions in the frequency-power mapping of the ACF parameter. The two PIN photodetectors (PD) are the optical-to-electrical conversion module. The DD-MZM internal structure is represented by two directional couplers (DC) with arbitrary coupling coefficients-the first DC (DC $)$ acts as an optical splitter-, two phase modulators (PM), and two phase shifters (PS). The PM can be fed, independently, by RF signals, $\mathrm{RF} 1$ and RF2, and the PS by distinct DC bias voltages, $\mathrm{BV}_{1}$ and $\mathrm{BV}_{2}$. This block diagram gives a global picture of a generalized IFM system aiming to obtain a power-to-frequency relationship, namely the ACF. To be effective, this parameter should be represented by a sharp curve as function of the input microwave frequency ${ }^{9}$.

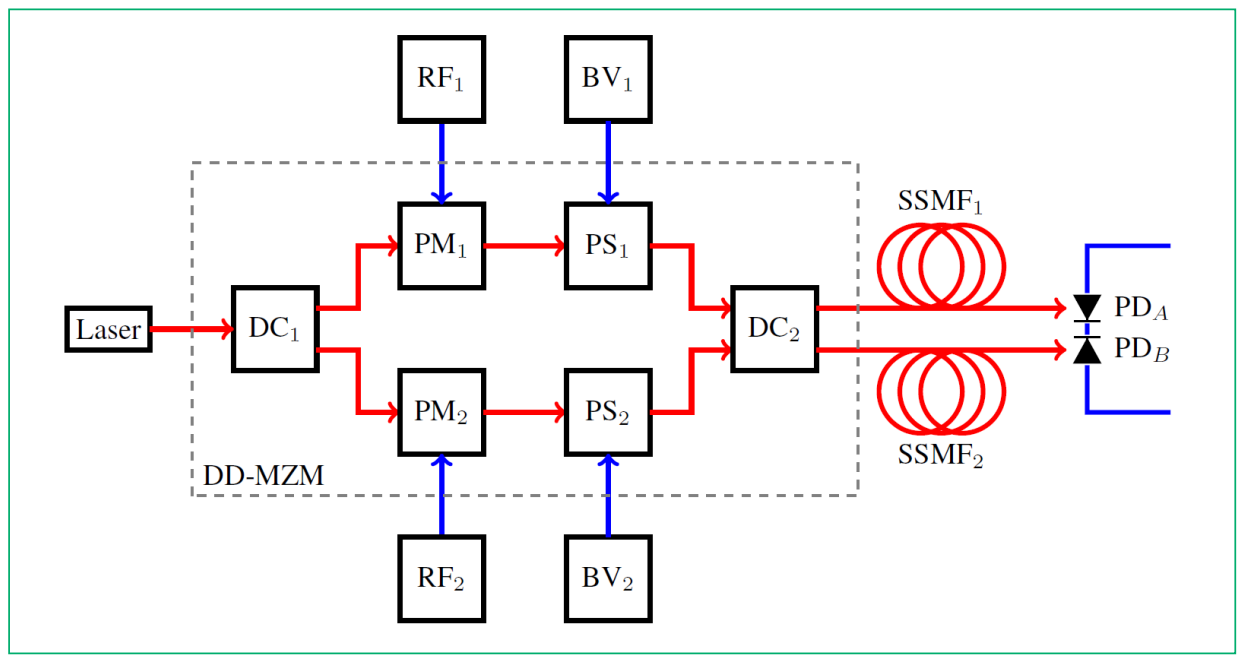

Figure 3: Functional block diagram of a typical instantaneous microwave frequency measurement system? RF: radio frequency signal; BV: bias voltage; DC: directional coupler; PM: phase modulator; PS: phase shifter; SSMF: standard single-mode fiber; PD: photodetector; DD-MZM: dual-drive Mach-Zehnder modulator.

\section{FORMULATION OF THE PROBLEM}

For a better understanding of the proposed device, Fig. 4 shows an enlarged view of the dual-output DD-MZM. In the DC1 block, $e^{(i n)}(t)=E^{(i n)} \cos \left(\omega_{o} t+\phi_{o}\right)$ is split according to the coupling coefficients $\varkappa_{1}$ and $\rho_{1}$. Usually, a $3 \mathrm{~dB}$ ratio is used, but here we have proposed the possibility of using arbitrary values of these coefficients, obeying the condition $\varkappa_{1}^{2}+\rho_{1}^{2}=1$, with $\mathrm{i}=1 ; 2$, by calculating the $\mathrm{DC}_{\mathrm{i}}$ scattering matrix ${ }^{10}$. These two new optical electric fields are modulated in the PM blocks by RF signals and biased in the PS blocks. These electric fields are recombined in the $\mathrm{DC}_{2}$ block according to its scattering matrix with coupling coefficients $\varkappa_{2}$ and $\rho_{2}$. In the proposed scheme, all these blocks are parameterizable.

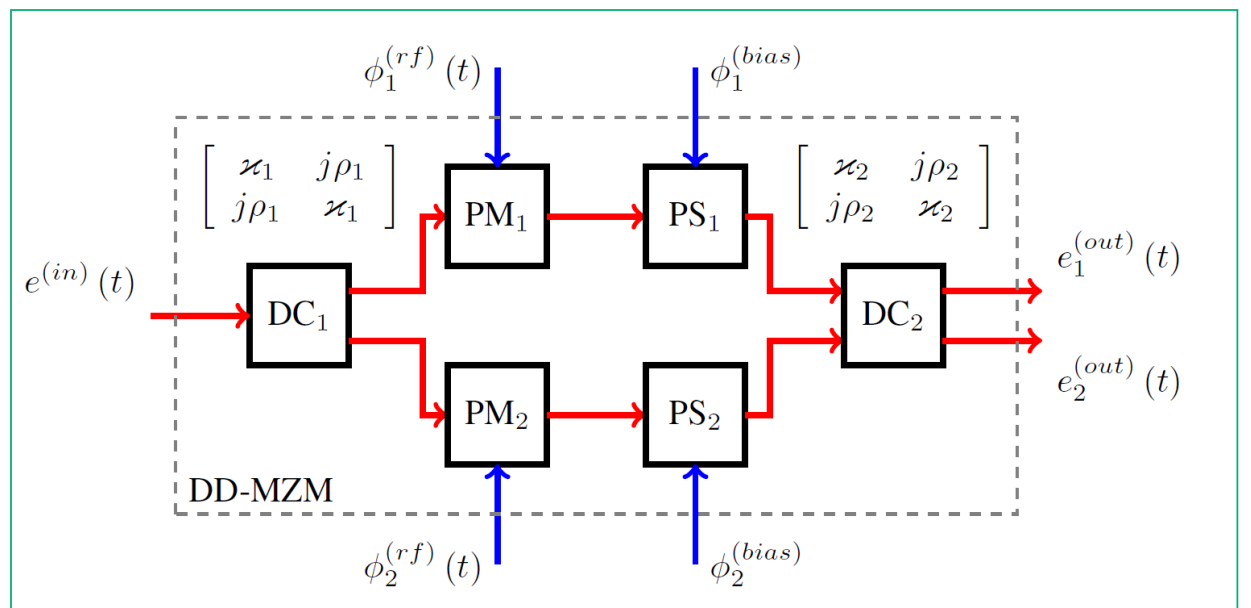

Figure 4: Schematic representation of a dual-output DD-MZM in terms of a functional block diagram with coupling coefficients scattering matrices. Electric fields $e_{1}{ }^{\text {(out) }}(t)$ and $e_{2}{ }^{\text {(out) }}(t)$ feed distinct optical fiber paths ${ }^{7}$.

DC: directional coupler; PM: phase modulator; PS: phase shifter; DD-MZM: dual-drive Mach-Zehnder modulator. 
It is emphasized that the dual-output DD-MZM permits some modulation formats, e.g., DSB, SSB and DSB suppressed carrier (DSB-SC). The SSB can be split in lower-sideband (LSB) and upper-sideband (USB) in the outputs 1 and 2, respectively. In this paper, only DSB and SSB were used, due to DSB-SC format to provide one output with odd order components and the other one with the even orders. This spectral characteristic is subject of a specific work.

These electric field outputs, $e_{1}{ }^{\text {(out) }}(t)$ and $e_{2}{ }^{\text {(out) }}(t)$, which obey the principle of energy conservation, feed distinct SSMF paths. Assuming $\bar{E}^{(i n)}=E^{(i n)} \mathrm{e}^{j \phi_{o}}$ as the complex optical electric field input envelope, and RF signals with the same frequency and amplitude feeding the PM1 and PM2, the DC2 output complex envelopes can be given by Carreira et al. (Eqs. 1 and 2):

$$
\begin{aligned}
& \bar{E}_{1}^{(\text {out })}=\Gamma \bar{E}^{(\text {in })} \sum_{n=-\infty}^{+\infty} a_{n}^{(1)} J_{n}(m) \mathrm{e}^{j n \omega_{r f} t} \\
& \bar{E}_{2}^{\text {(out })}=j \Gamma \bar{E}^{(\text {in })} \sum_{n=-\infty}^{+\infty} a_{n}^{(2)} J_{n}(m) e^{j n \omega_{r f} t}
\end{aligned}
$$

in which: $\Gamma=$ the insertion power loss of the link; $\mathrm{J}_{\mathrm{n}}=$ the $\mathrm{n}$-th order Bessel function of the first kind; $\mathrm{m}=\pi \mathrm{V}_{\mathrm{rf}} / \mathrm{V}_{\pi}=$ the modulation index, being $\mathrm{V}_{\mathrm{rf}}$ the RF signal amplitude and $\mathrm{V}_{\pi}$ the DD-MZM half-wave voltage. The complex coefficients $\mathrm{a}_{\mathrm{n}}{ }^{(1)}$ and $\mathrm{a}_{\mathrm{n}}{ }^{(2)}$ are expressed as Eqs. 3 and 4 :

$$
\begin{aligned}
& a_{n}^{(1)}=\varkappa_{1} \varkappa_{2} \mathrm{e}^{j\left(n \phi_{1}^{(0)}+\phi_{1}^{(\text {bias })}\right)}-\rho_{1} \rho_{2} \mathrm{e}^{j\left(n \phi_{2}^{(0)}+\phi_{2}^{(\text {bias })}\right)} \\
& a_{n}^{(2)}=\varkappa_{1} \rho_{2} \mathrm{e}^{j\left(n \phi_{1}^{(0)}+\phi_{1}^{(\text {bias })}\right)}+\varkappa_{2} \rho_{1} \mathrm{e}^{j\left(n \phi_{2}^{(0)}+\phi_{2}^{(\text {bias })}\right)}
\end{aligned}
$$

in which: $\phi_{i}^{(0)}=$ the PM RF signal initial phase; $\phi_{i}^{(\text {bias })}=$ the DC bias phase shift introduced by the PS .

The output signals travel through optical fiber paths with lengths $\mathrm{L}_{1}$ and $\mathrm{L}_{2}$ and then are detected by $\mathrm{PD}_{\mathrm{A}}$ and $\mathrm{PD}_{\mathrm{B}}$. At this point, the chromatic dispersion and attenuation effects of each fiber path are considered. Thus, the complex envelopes incident on $\mathrm{PD}_{\mathrm{A}}$ and $\mathrm{PD}_{\mathrm{B}}$ are expressed in Eqs. 5 and 6:

$$
\begin{aligned}
& \bar{E}_{A}=\bar{E}_{1}^{(\text {out })} \mathrm{e}^{-\gamma\left(\omega_{o}+n \omega_{r f}\right) L_{1}} \\
& \bar{E}_{B}=\bar{E}_{2}^{\text {(out })} \mathrm{e}^{-\gamma\left(\omega_{o}+n \omega_{r f}\right) L_{2}}
\end{aligned}
$$

in which: $\gamma=$ the fiber-optic propagation constant.

The photocurrent in each PD is given by Equation 7:

$$
I_{A, B}(t)=\frac{\Re_{A ; B}}{2 \eta}\left\langle\left|e_{A, B}^{(\text {out })}(t)\right|^{2}\right\rangle \xi
$$

in which: the bracketed term $=$ the time average of $\left|e_{A, B}^{(o u t)}(t)\right|^{2}$ over an optical cycle; $\mathrm{A} ; \mathrm{B}=$ each $\mathrm{PD} ; \mathfrak{R}_{A ; B}=$ the PD responsivities; $\xi=$ the PD effective cross sections; $\eta=$ the optical wave impedance.

Expanding $\gamma$ in Taylor series and substituting Eqs. 5 and 6 in Eq. 7, one takes:

$$
\begin{aligned}
& I_{A}(t)=\Re_{A} \Gamma^{2} P^{(i n)} \mathrm{e}^{-2 \alpha_{1} L_{1}} \sum_{n=-\infty}^{+\infty} j^{n} \mathrm{e}^{j n \omega_{r f} t} \times\left\{\mathcal{\varkappa}_{1}^{2} \mathcal{\varkappa}_{2}^{2} \mathrm{e}^{j n \Delta \phi_{r f}} J_{n}\left[-2 m \sin \left(\Phi_{1}\right)\right]+\rho_{1}^{2} \rho_{2}^{2} J_{n}\left[-2 m \sin \left(\Phi_{1}\right)\right]+\right. \\
& \left.-\varkappa_{1} \mathcal{\varkappa}_{2} \rho_{1} \rho_{2} \mathrm{e}^{j\left(\frac{n \Delta \phi_{r f}}{2}+\Delta \phi_{\text {bias }}\right)} J_{n}\left[2 m \sin \left(\frac{\Delta \phi_{r f}}{2}-\Phi_{1}\right)\right]-\varkappa_{1} \mathcal{\varkappa}_{2} \rho_{1} \rho_{2} \mathrm{e}^{j\left(\frac{n \Delta \phi_{r f}}{2}-\Delta \phi_{\text {bias }}\right)} J_{n}\left[-2 m \sin \left(\frac{\Delta \phi_{r f}}{2}+\Phi_{1}\right)\right]\right\} \\
& I_{B}(t)=\mathfrak{R}_{B} \Gamma^{2} P^{(i n)} e^{-2 \alpha_{2} L_{2}} \sum_{n=-\infty}^{+\infty} j^{n} e^{j n \omega_{r f} t} \times\left\{\mathcal{\varkappa}_{1}^{2} \rho_{2}^{2} e^{j n \Delta \phi_{r f}} J_{n}\left[-2 m \sin \left(\Phi_{2}\right)\right]+\varkappa_{2}^{2} \rho_{1}^{2} J_{n}\left[-2 m \sin \left(\Phi_{2}\right)\right]+\right. \\
& \left.+\varkappa_{1} \mathcal{\varkappa}_{2} \rho_{1} \rho_{2} e^{j\left(\frac{n \Delta \phi_{r f}}{2}+\Delta \phi_{\text {bias }}\right)} J_{n}\left[2 m \sin \left(\frac{\Delta \phi_{r f}}{2}-\Phi_{2}\right)\right]+\varkappa_{1} \mathcal{\varkappa}_{2} \rho_{1} \rho_{2} e^{j\left(\frac{n \Delta \phi_{r f}}{2}-\Delta \phi_{\text {bias }}\right)} J_{n}\left[-2 m \sin \left(\frac{\Delta \phi_{r f}}{2}+\Phi_{2}\right)\right]\right\}
\end{aligned}
$$

in which: $\Delta \phi_{\text {bias }}=$ the DD-MZM DC bias phase shift; $\Delta \phi_{\mathrm{rf}}=$ the RF phase change set in a hybrid; $\alpha_{1 ; 2}=$ the optical fiber attenuations; $\Phi_{1 ; 2}=\beta_{2} \omega_{r f}^{2} n L_{1 ; 2} / 2, \beta_{2}=-D_{1 ; 2} \lambda_{o}^{2} / 2 \pi c=$ the second-order dispersion coefficient; $\mathrm{D}_{1 ; 2}=$ the fiber dispersion parameters; $\lambda_{\mathrm{o}}=$ the laser wavelength; $c=$ the speed of light in vacuum.

The Graf's addition theorem was used to calculate the current waveform in each PD, enabling a model not limited to small signals, thus allowing the consideration of high-modulation indexes ${ }^{11}$. Likewise, the ACF can be obtained for DSB and SSB modulation formats thanks to the use of a dual-output DD-MZM, which makes the system tunable. The semi-analytical model developed also includes higher order lateral bands, enabling ACF ratios for spectral components of orders different than unity. 
For a proper discussion, the DD-MZM is set for a SSB modulation format $\left(\Delta \phi_{\text {bias }}=\pi / 2\right.$ and $\left.\Delta \phi_{\mathrm{rf}}=\pi / 2\right)$, the DC coupling coefficients are set for $3 \mathrm{~dB}$ and the first harmonic component is selected $(\mathrm{n}=1)$. Therefore, from Equations 8 and 9 , one can obtain the instantaneous RF power output $P_{r f}^{(A ; B)}(t)=I_{A ; B}(t)^{2} Z_{A ; B}$ (Eqs. 10 and 11) for each $\mathrm{PD}$, in which $\mathrm{Z}_{\mathrm{A} ; \mathrm{B}}$ is the $\mathrm{PD}_{\mathrm{A} ; \mathrm{B}}$ impedance:

$$
\begin{aligned}
& P_{A}(t)=A_{A}^{2}\left[B_{A}^{2}+C_{A}^{2}+\left(B_{A}^{2}-C_{A}^{2}\right) \sin (2 \omega t)+2 B_{A} C_{A} \cos (2 \omega t)\right] \\
& P_{B}(t)=A_{B}^{2}\left[B_{B}^{2}+C_{B}^{2}+\left(B_{B}^{2}-C_{B}^{2}\right) \sin (2 \omega t)-2 B_{B} C_{B} \cos (2 \omega t)\right]
\end{aligned}
$$

in which the coefficients $\mathrm{A}_{\mathrm{A} ; \mathrm{B}}, \mathrm{B}_{\mathrm{A} ; \mathrm{B}}$, and $\mathrm{C}_{\mathrm{A} ; \mathrm{B}}$ can be expressed as Eqs. 12, 13 and 14:

$$
\begin{gathered}
A_{A ; B}=\frac{\Re_{A ; B}}{4} \Gamma^{2} P^{(i n)} e^{-2 \alpha_{1 ; 2} L_{1 ; 2} Z_{A ; B}} \\
B_{A ; B}=J_{1}\left[2 m \sin \left(\Phi_{1 ; 2}\right)\right] \\
C_{A ; B}=\frac{\sqrt{2}}{2}\left\{J_{1}\left[m \sqrt{2}\left(\cos \Phi_{1 ; 2}-\sin \Phi_{1 ; 2}\right)\right]+J_{1}\left[m \sqrt{2}\left(\cos \Phi_{1 ; 2}+\sin \Phi_{1 ; 2}\right)\right]\right\}
\end{gathered}
$$

Equations 10 and 11 show terms related to active and reactive power, cosine and sine, respectively, that will be discussed in future works. Here we are interested in the instantaneous $A C F$, which is defined by the ratio between the RF power obtained in $\mathrm{PD}_{\mathrm{A}}$ and $\mathrm{PD}_{\mathrm{B}}^{4,12}$. Thus, assuming the same fiber lengths $\left(\mathrm{L}_{1}=\mathrm{L}_{2}\right)$, dispersion parameters $\left(\mathrm{D}_{1}=\mathrm{D}_{2}\right)$, PD responsivities $\left(\Re_{\mathrm{A}}=\Re_{\mathrm{B}}\right)$ and impedances $\left(\mathrm{Z}_{\mathrm{A}}=\mathrm{Z}_{\mathrm{B}}\right)$, at instant $\mathrm{t}=\pi / 2 \omega_{\mathrm{rf}}$, one obtains Equation 15 :

$$
A C F=\frac{P_{A}(t)}{P_{B}(t)}=\left(\frac{B-C}{B+C}\right)^{2}
$$

Equation 15, as far as we know, is not represented in the literature. It allows to reproduce previously published results and provides new ones, e.g., the impact of high-modulation indexes.

\section{ANALYTICAL AND SIMULATION RESULTS}

For the analytical analysis, it is assumed $\mathrm{m}=0.2$ (low-modulation index), $\mathrm{L}=20 \mathrm{~km}, \mathrm{D}=17 \mathrm{ps} / \mathrm{km} . \mathrm{nm}$ and $\lambda_{\mathrm{o}}=1,550 \mathrm{~nm}$ in Eq. 15 . Figure 5 shows $\mathrm{PD}_{\mathrm{A}}$ and $\mathrm{PD}_{\mathrm{B}} \mathrm{RF}$ power outputs, normalized with respect to the maximum value of $P_{r f}^{(B)}$, and ACF. Note that, from Eq. $15, P_{r f}^{(A)}(t)$ and $P_{r f}^{(B)}(t)$ are the active powers at the instant $\mathrm{t}=\pi / 2 \omega_{\text {rf }}$. Furthermore, Fig. 5 reproduces Li et al's work ${ }^{5}$ even with both PM fed by RF signals.

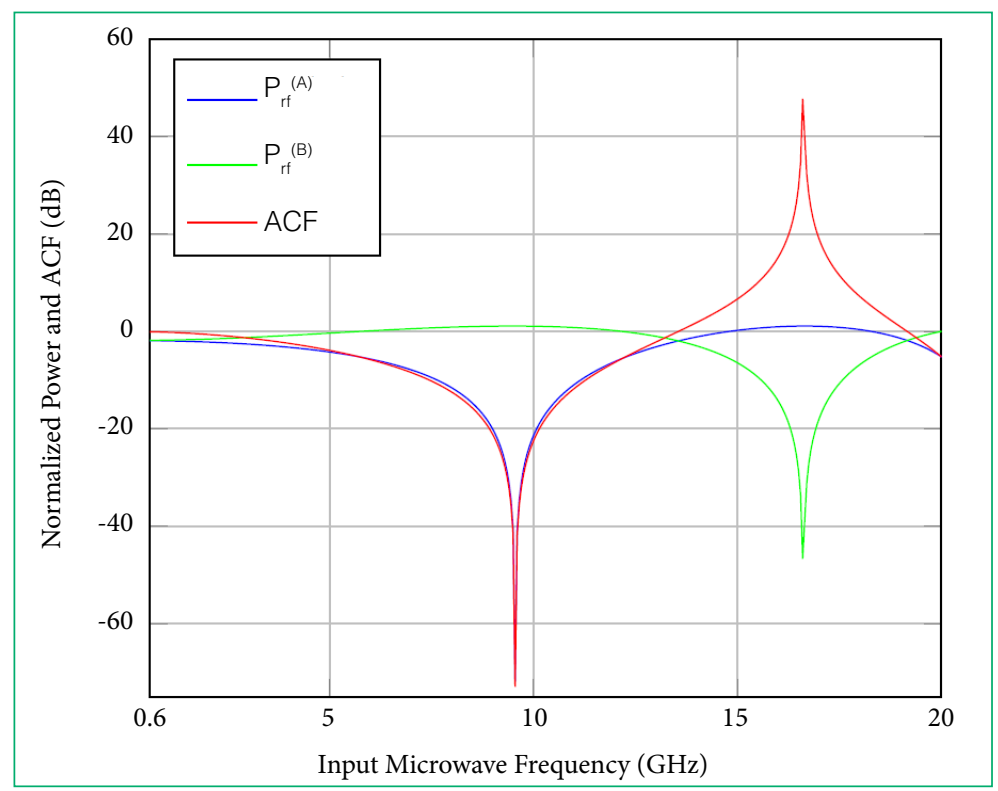

Figure 5: $\mathrm{PD}_{\mathrm{A}}$ and $\mathrm{PD}_{\mathrm{B}} \mathrm{RF}$ powers, normalized with respect to the maximum value of $\mathrm{P}_{\mathrm{rf}}^{(\mathrm{B})}$, and $\mathrm{ACF}$ for modulation format $\mathrm{SSB}$, modulation index $m=0.2$, optical fiber path $1 \mathrm{~L}_{1}=20 \mathrm{~km}$, optical fiber path $2 \mathrm{~L}_{2}=20 \mathrm{~km}$ and $\mathrm{DC}_{1}$ and $\mathrm{DC}_{2}$ coupling coefficients set with $3 \mathrm{~dB}$.

ACF: amplitude comparison function; PD: photodetector; RF: radio frequency; SSB: single-sideband modulation; DC: directional couplers. 
For the simulation analysis, the OptiSystem 17.0 was set with the same parameter values as the analytical analysis. Figure 6 presents both the analytical and simulation results, showing good agreement. The lowest ACF value is $-67.99 \mathrm{~dB}$ at $9.69 \mathrm{GHz}$ for the simulation, while Eq. 15 gives $-64.14 \mathrm{~dB}$ at $9.56 \mathrm{GHz}$. It represents an ACF difference of $3.85 \mathrm{~dB}$ and a frequency difference of $130 \mathrm{MHz}$. The highest $\mathrm{ACF}$ value is $52.35 \mathrm{~dB}$ at $16.82 \mathrm{GHz}$ for the simulation, while it is $54.93 \mathrm{~dB}$ at $16.61 \mathrm{GHz}$ for the analytical. It represents a difference in ACF of $2.58 \mathrm{~dB}$ and in frequency of $210 \mathrm{MHz}$.

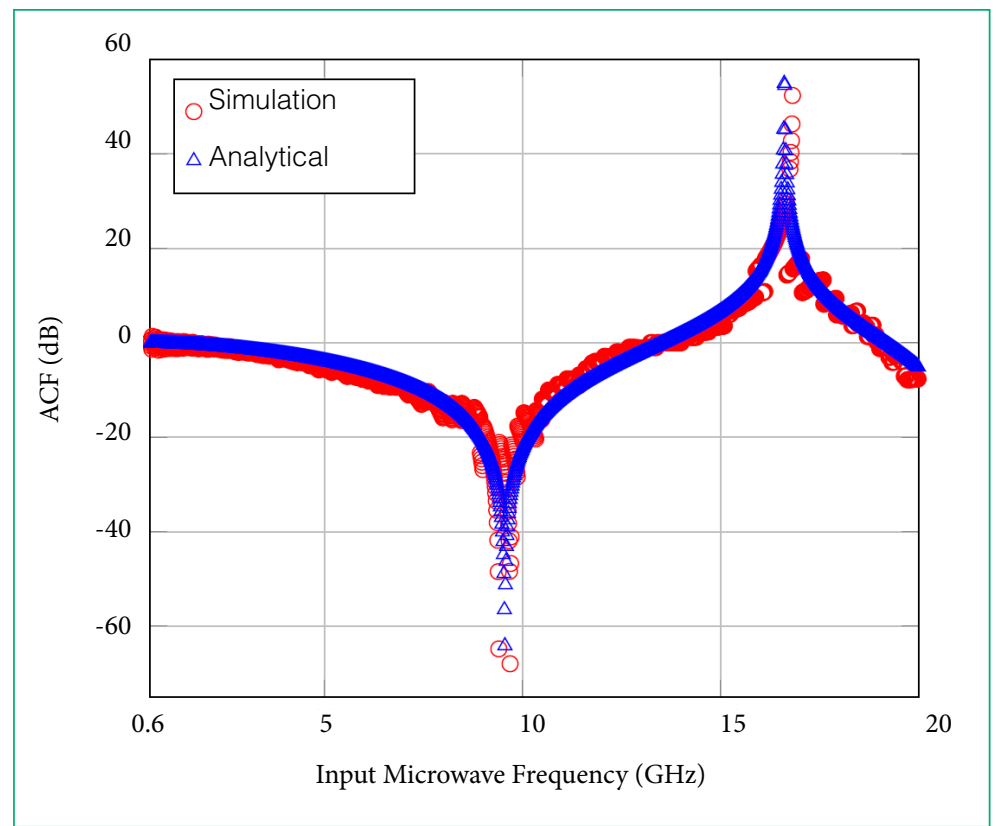

Figure 6: Simulation and analytical results for amplitude comparison function (ACF). The lowest value differences are $3.85 \mathrm{~dB}$ for the $\mathrm{ACF}$ and $130 \mathrm{MHz}$ for the frequency. The highest value differences are $2.58 \mathrm{~dB}$ for the $\mathrm{ACF}$ and $210 \mathrm{MHz}$ for the frequency.

As discussed in the previous section, Eqs. 8 and 9 allows flexibility to vary other parameters, e.g., DC coupling coefficients, fiber length, modulation index and modulation formats. In this work, we were interested in a SSB approach with low-modulation index, same fiber length and $3 \mathrm{~dB}$ DC coupling coefficients for comparison of analytical and simulation results with those ones published in the literature. The variation of these other parameters and other possible contributions to cognitive radio will be subject of future works.

\section{CONCLUSION}

In this work, we have discussed a photonic-assisted microwave frequency measurement towards cognitive radio. The proposed device permits some parameter adjustments, namely DC coupling coefficients, fiber length, modulation index and modulation formats, what makes the system tunable. As far as we know, this work brings a novel and complete analytical model not clearly presented in the literature. It reproduces previously published results, besides a good agreement with the simulation result. The frequency differences between the analytical and simulation results are $130 \mathrm{MHz}$ and $210 \mathrm{MHz}$ for the lowest and highest ACF values, respectively. The authors emphasize that this is not a final work, but part of an ongoing research. It is advancing towards to incorporate coherent detection in the scheme and optical fiber nonlinearities.

\section{ACKNOWLEDGMENT}

The authors would like to thank the Marinha do Brasil and the Força Aérea Brasileira for the support.

\section{REFERENCES}

1. Zhu D, Pan S. Broadband cognitive radio enabled by photonics. J Light Technol. 2020;38(12):3076-88. https://doi.org/10.1109/JLT.2020.2993021

2. East PW. Fifty years of instantaneous frequency measurement. IET Radar Sonar Nav. 2012;6(2):112-22. https://doi.org/10.1049/ietrsn.2011.0177

3. Li Y, Pei L, Li J, Zheng J, Wang Y, Yuan J, et al. Instantaneous microwave frequency measurement with improved resolution. Opt Commun. 2015;354:140-7. https://doi.org/10.1016/j.optcom.2015.05.050 
4. Zhou J, Fu S, Aditya S, Shum PP, Lin C. Instantaneous microwave frequency measurement using photonic technique. IEEE Photonics Tech L. 2009;21(15):1069-71. https://doi.org/10.1109/LPT.2009.2022637

5. Li J, Fu S, Xu K, Zhou JQ, Shum P, Wu J, et al. Photonic-assisted microwave frequency measurement with higher resolution and tunable range. Opt Lett. 2009;34(6):743-5. https://doi.org/10.1364/OL.34.000743

6. Bui LA. Recent advances in microwave photonics instantaneous frequency measurements. Prog Quantum Electron. 2020;69:100237. https:// doi.org/10.1016/j.pquantelec.2019.100237

7. Carreira RR, Barroso JJ, Oliveira JEB, Gonçalves AP, Santos AR, Coutinho OL, et al. Generalized analysis of a tunable range photonic-assisted instantaneous frequency measurement of microwave signals. in-press.

8. Zou X, Lu B, Pan W, Yan L, Stöhr A, Yao J. Photonics for microwave measurements. Laser Photonics Rev. 2016;10(5):711-34. https://doi. org/10.1002/lpor.201600019

9. Zou X, Chi H, Yao J. Microwave frequency measurement based on optical power monitoring using a complementary optical filter pair. IEEE Trans Microw Theory Tech. 2009;57(2):505-11. https://doi.org/10.1109/TMTT.2008.2011237

10. Rediker RH, Leonbercer FJ. Analysis of integrated-optics near $3 \mathrm{~dB}$ coupler and mach-zehnder interferometric modulator using fourport scattering matrix. IEEE Trans Microw Theory Tech. 1982;30(10):1801-4. https://doi.org/10.1109/TMTT.1982.1131324

11. Carreira RR, Barroso JJ, Oliveira JEB. Mitigation of chromatic dispersion in fiber-optic link using dual-drive mach-zehnder modulator and heterodyne detection technique. In: SBMO/IEEE MTTS International Microwave and Optoelectronics Conference (IMOC), 2019. IEEE; 2019.

12. Xu K, Dai J, Duan R, Dai Y, Li Y, Wu J, et al. Instantaneous microwave frequency measurement based on phase-modulated links with interferometric detection. IEEE Photonic Tech L. 2011;23(18):1328-30. https://doi.org/10.1109/LPT.2011.2160336 